\title{
EDGE NUMBER RESULTS FOR PIECEWISE-LINEAR KNOTS
}

\author{
MONICA MEISSEN \\ Bethel College, North Newton, Kansas 67117, U.S.A. \\ E-mail:meissen@bethelks.edu
}

\begin{abstract}
The minimal number of edges required to form a knot or link of type $\mathrm{K}$ is the edge number of $\mathrm{K}$, and is denoted $\mathrm{e}(\mathrm{K})$. When knots are drawn with edges, they are appropriately called piecewise-linear or PL knots. This paper presents some edge number results for PL knots. Included are illustrations of and integer coordinates for the vertices of several prime PL knots.
\end{abstract}

1. Introduction. An elementary piecewise-linear knot invariant is the edge number $\mathrm{e}(\mathrm{K})$ of $\mathrm{K}$, which is the minimal number of edges required to form the given knot or link $\mathrm{K}$. It is already known $[4,6,7,9]$ that the edge numbers of prime knots $^{1}$ are given as follows:

$$
e(\text { unknot })=3, \quad e\left(3_{1}\right)=6, \quad e\left(4_{1}\right)=7
$$

$$
e(K)=8 \text { for } K=5_{1}, 5_{2}, 6_{1}, 6_{2} \text {, or } 6_{3} \text { and } e(K) \geq 8 \text { for any other knot. }
$$

The minimal number of edges required to form some links and composite knots is also known $[1,4,5]$. For example, a composition of $n$ trefoils has an edge number of $2 n+4$ [1]. Exact edge number results are difficult to obtain. In fact, edge numbers have not been determined in general for the most basic types of knots, the torus knots. Lower and upper bounds have been determined for torus knots, which together with certain additional hypotheses, yield exact results in these special cases [4, 5].

The purpose of this paper is to present edge number results which were obtained using a program called KED [3] written by K. Hunt as part of his thesis research in computer science at the University of Iowa. Each one of the several drawings of prime knots provided in Section 3 were produced using a similar type of program called MING [10], written by Professor Y-Q. Wu.

The work presented here was completed while at The University of Iowa.

The paper is in final form and no version of it will be published elsewhere.

${ }^{1}$ Notation used in this paper is adopted from [8]. 
2. Edge number results. Since exact edge number results had previously been obtained for all prime knots with crossing number less than seven $[4,6,7,9]$, the prime knots with a crossing number of seven were logically the next ones to be considered. These knots were all constructed using only nine edges, therefore the result $(\dagger)$ stated in the introduction implies that each must have an edge number equal to either 8 or 9 . A few knots with crossing number 8 were also realized, specifically the non-alternating ones, since these were suspected to have smaller edge numbers. $8_{19}$ and $8_{20}$ were drawn with eight edges ${ }^{2}$ and $8_{21}$ was constructed from nine edges. Therefore, $e\left(8_{19}\right)=8=e\left(8_{20}\right)$ and $e\left(8_{21}\right)=8$ or 9 .

Each of the knots whose illustrations appear in Section 3 were obtained using KED [3], which gives the coordinates of the vertices of each of the knots produced as numbers with 16 decimal places. "Nice" integer coordinates were more desirable. In the table provided in Section 3, notice that each of the knots was, in fact, realized with integer coordinates $(x, y, z)$ such that $-10 \leq x, y, z \leq 10$. These were obtained by examining the coordinates of each knot individually, manually inputting the "nice" integer coordinates nearby the original ones, and then inspecting the result to check that the knot type was preserved in the process. In a few cases, the coordinates had to be adjusted again in order to maintain the same knot type as the original.

3. Prime knots with integer coordinates. This section contains illustrations of and integer coordinates for the prime knots with crossing number less than 8 . Three nonalternating knots with crossing number eight are also included, as well as a symmetry presentation for the figure-eight knot $4_{1}$. The drawings provided were produced by MING [10] using the cylinder-ball rendering and by choosing the knot color to be grey. In MING, there is also an option to obtain an image of the knot as it would be seen "viewed from above". In other words, the image has been projected down to the xy-plane, but in such a way that the crossings are still discernible. This option was selected, thereby producing illustrations which are easily interpreted. For example, the $3_{1}$ knot shown below has leftmost vertex 5 and rightmost vertex 6 .

\begin{tabular}{|c|c|c|c|c|}
\hline Knot & Integer coordin & es of & he vertices & MING illustration \\
\hline $3_{1}$ & 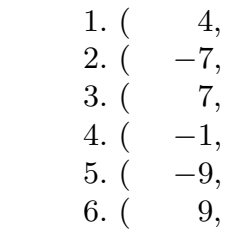 & $\begin{array}{r}9, \\
-7, \\
-9, \\
9, \\
-3, \\
-5,\end{array}$ & $\begin{array}{r}5) \\
-5) \\
5) \\
-5) \\
5) \\
-5)\end{array}$ & \\
\hline
\end{tabular}

\footnotetext{
${ }^{2}$ K. Millett (personal communication) has realized $8_{20}$ with 8 edges of equal length.
} 


\begin{tabular}{|c|c|c|c|c|}
\hline Knot & \multicolumn{3}{|c|}{ Integer coordinates of the vertices } & MING Illustration \\
\hline $4_{1}$ & 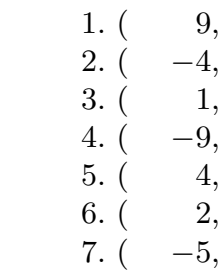 & $\begin{array}{r}-6, \\
-7, \\
7, \\
2, \\
-5, \\
2, \\
2,\end{array}$ & $\begin{array}{r}3) \\
3) \\
2) \\
-10) \\
10) \\
-2) \\
5)\end{array}$ & \\
\hline
\end{tabular}

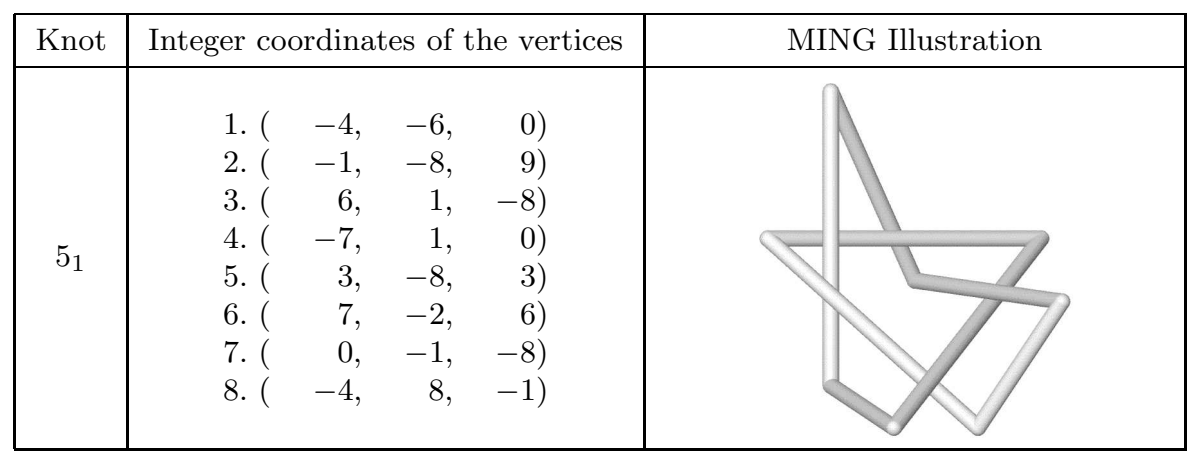

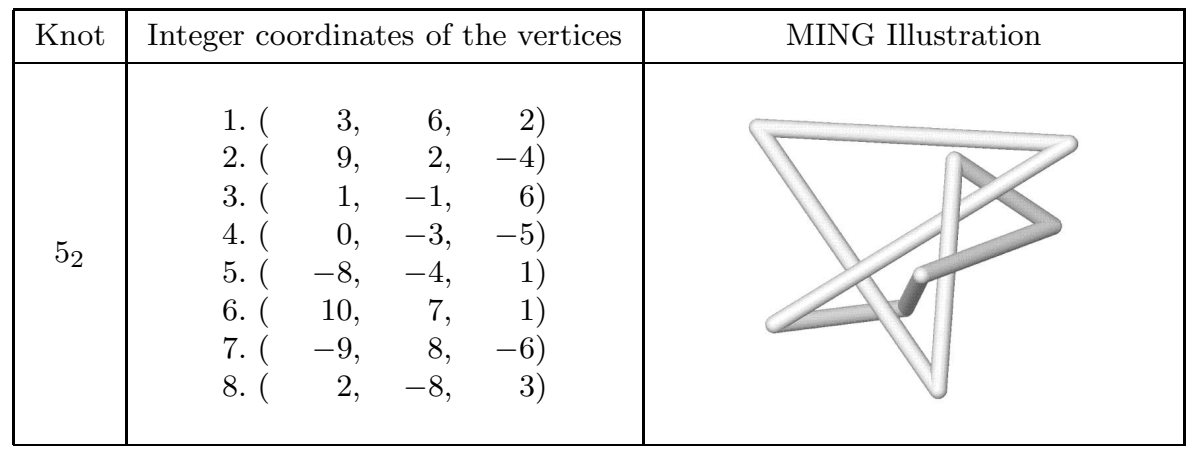

\begin{tabular}{|c|c|c|c|}
\hline Knot & Integer coordinat & tes of the vertices & MING Illustration \\
\hline 61 & 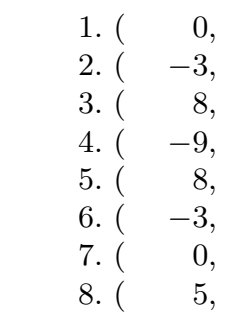 & $\begin{array}{rr}-8, & -5) \\
-7, & 3) \\
10, & -4) \\
0, & 5) \\
-10, & -5) \\
7, & 6) \\
8, & -5) \\
0, & 5)\end{array}$ & \\
\hline
\end{tabular}




\begin{tabular}{|c|c|c|c|c|}
\hline Knot & Integer coordin & es of & e vertices & MING Illustration \\
\hline $6_{2}$ & 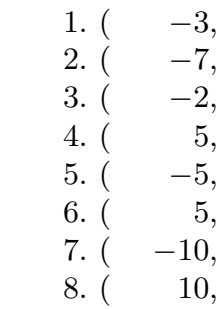 & $\begin{array}{r}-10, \\
-8, \\
-1, \\
4, \\
10, \\
-10, \\
-4, \\
9,\end{array}$ & $\begin{array}{r}7) \\
-10) \\
10) \\
-7) \\
-2) \\
10) \\
-6) \\
-1)\end{array}$ & \\
\hline
\end{tabular}

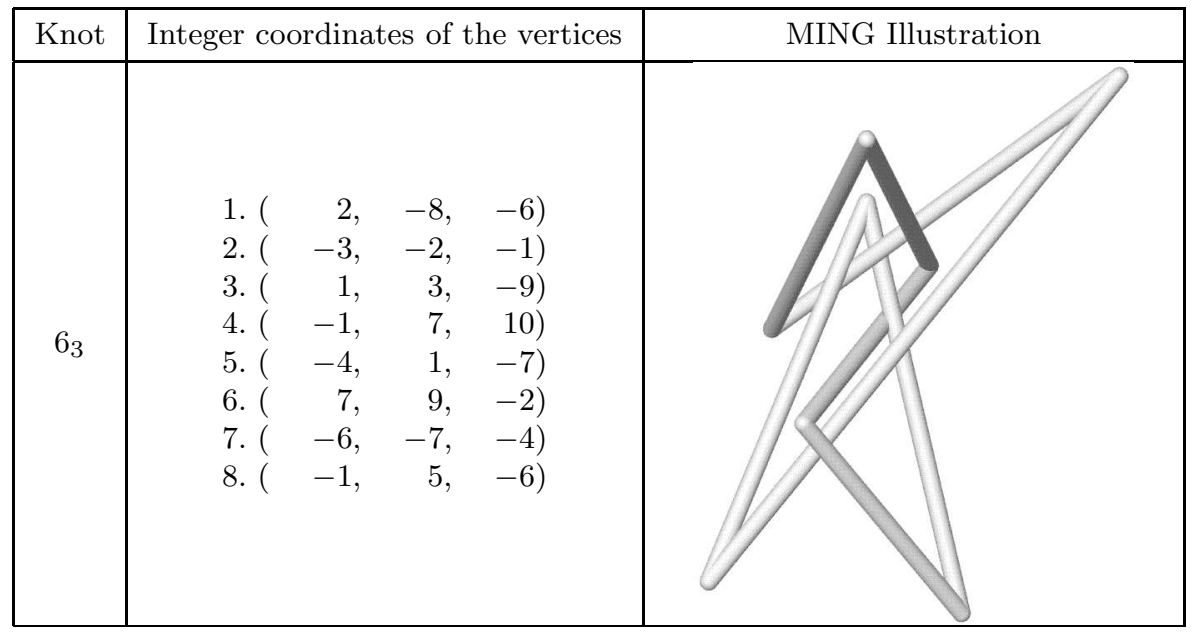

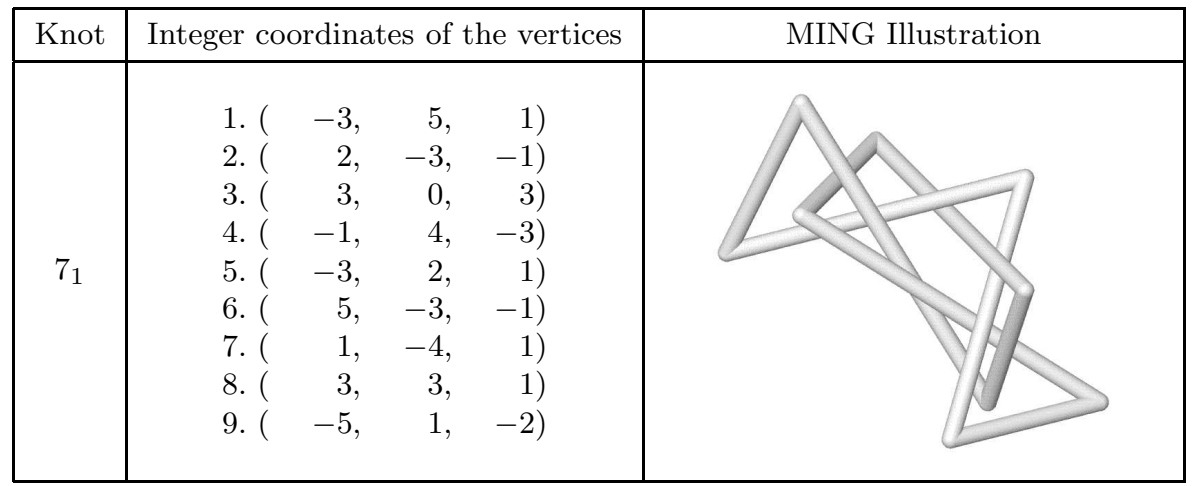




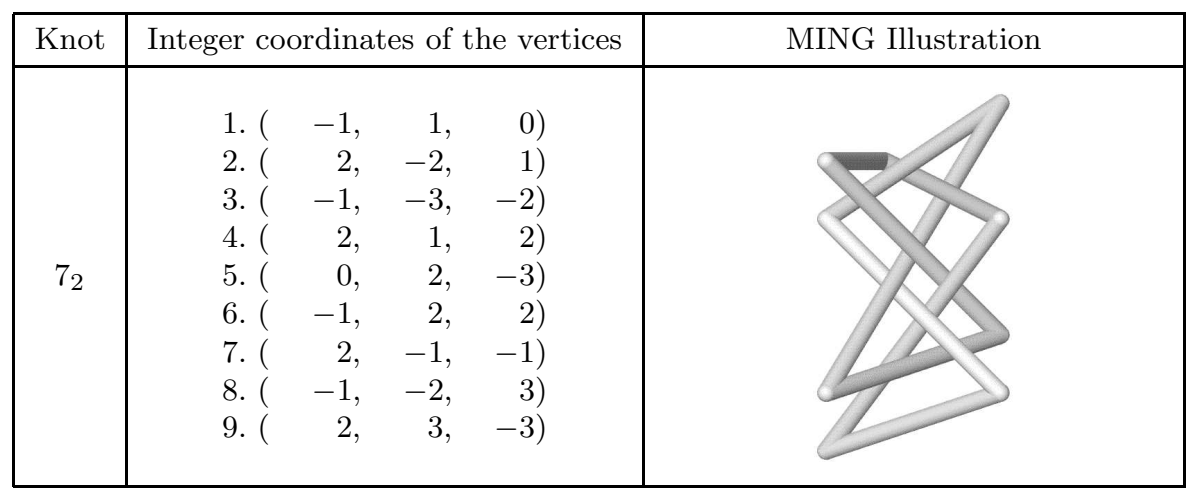

\begin{tabular}{|c|c|c|c|}
\hline Knot & Integer coordin & es of the vertices & MING Illustration \\
\hline $7_{3}$ & 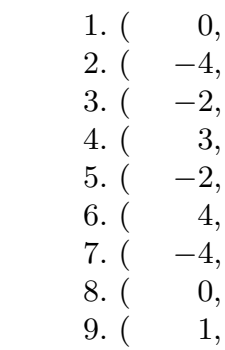 & $\begin{array}{rr}3, & -2) \\
-1, & 1) \\
-1, & -2) \\
-4, & 4) \\
5, & -3) \\
2, & 1) \\
1, & 0) \\
-4, & -1) \\
1, & 3)\end{array}$ & \\
\hline
\end{tabular}

\begin{tabular}{|c|c|c|c|}
\hline Knot & Integer coordina & es of the vertices & MING Illustration \\
\hline $7_{4}$ & 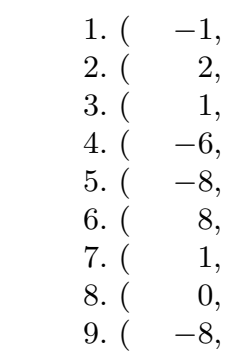 & $\begin{array}{rr}1, & 1) \\
6, & 5) \\
-6, & 2) \\
5, & 2) \\
4, & -1) \\
-1, & 6) \\
2, & 5) \\
5, & -5) \\
3, & 4)\end{array}$ & \\
\hline
\end{tabular}

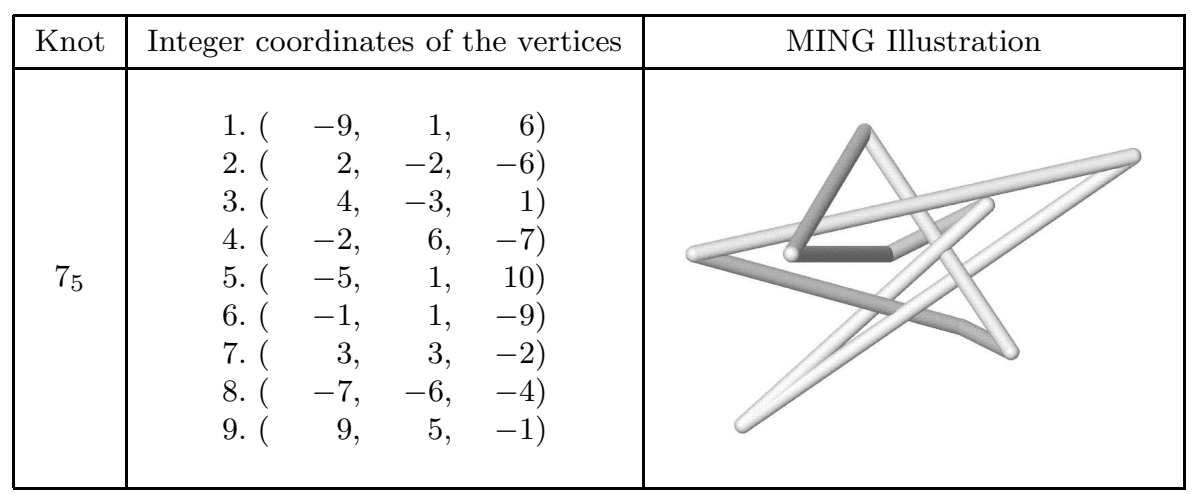




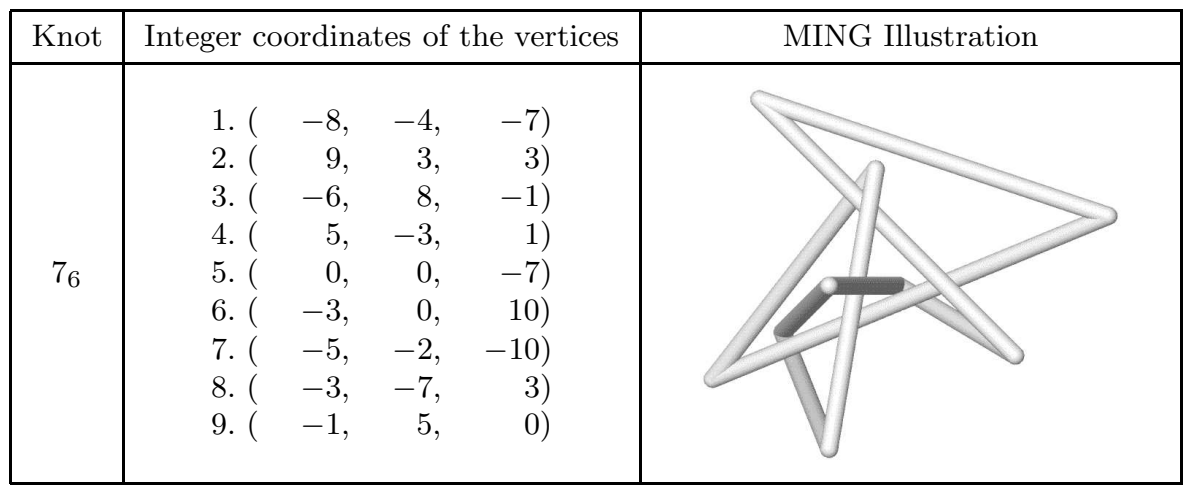

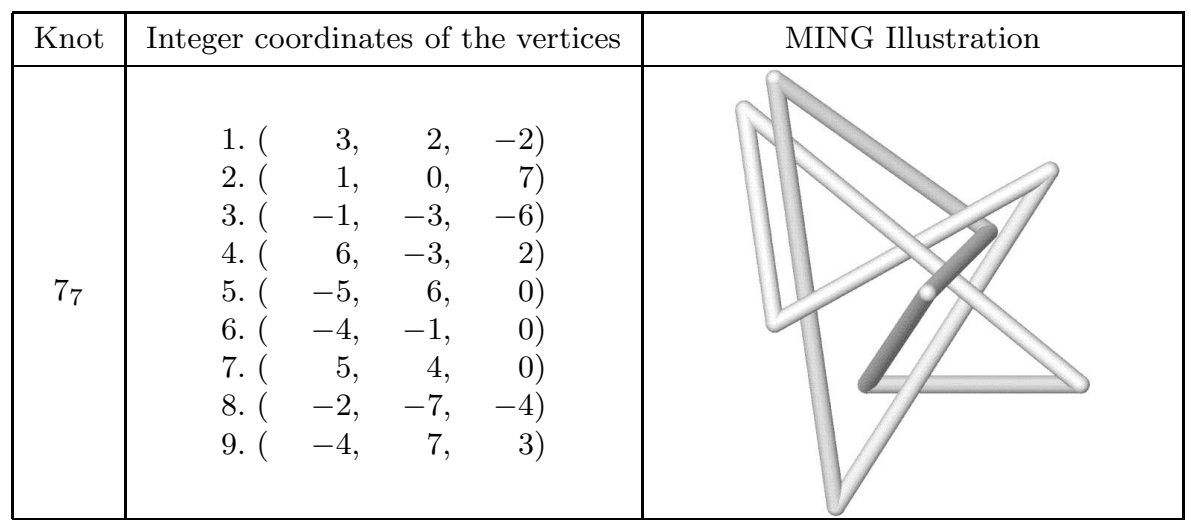

\begin{tabular}{|c|c|c|c|}
\hline Knot & Integer coordinates of & he vertices & MING Illustration \\
\hline 819 & 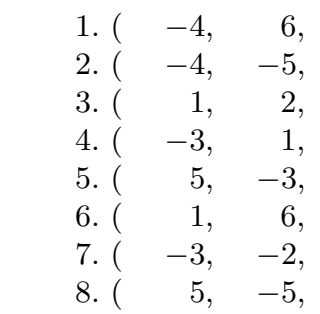 & $\begin{array}{r}5) \\
3) \\
3) \\
2) \\
7) \\
-10) \\
10) \\
0)\end{array}$ & \\
\hline
\end{tabular}

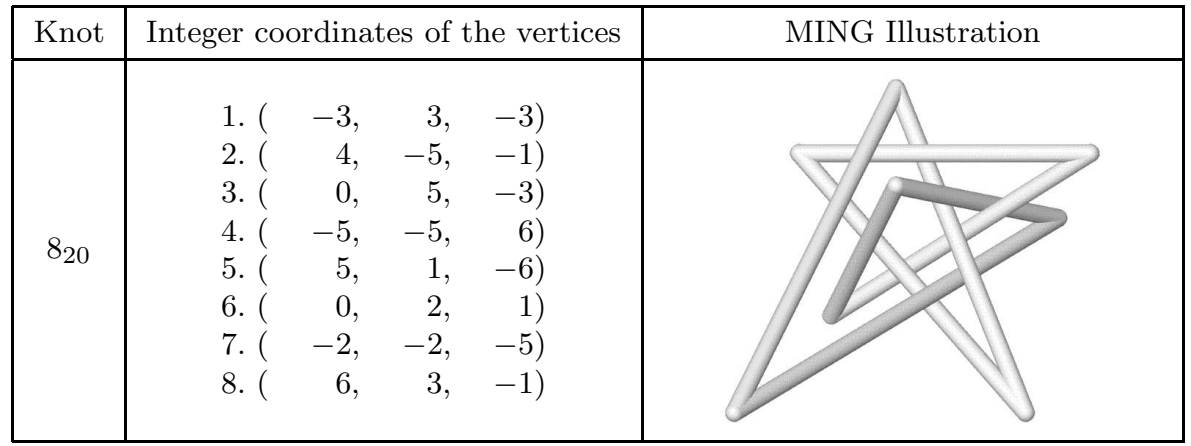




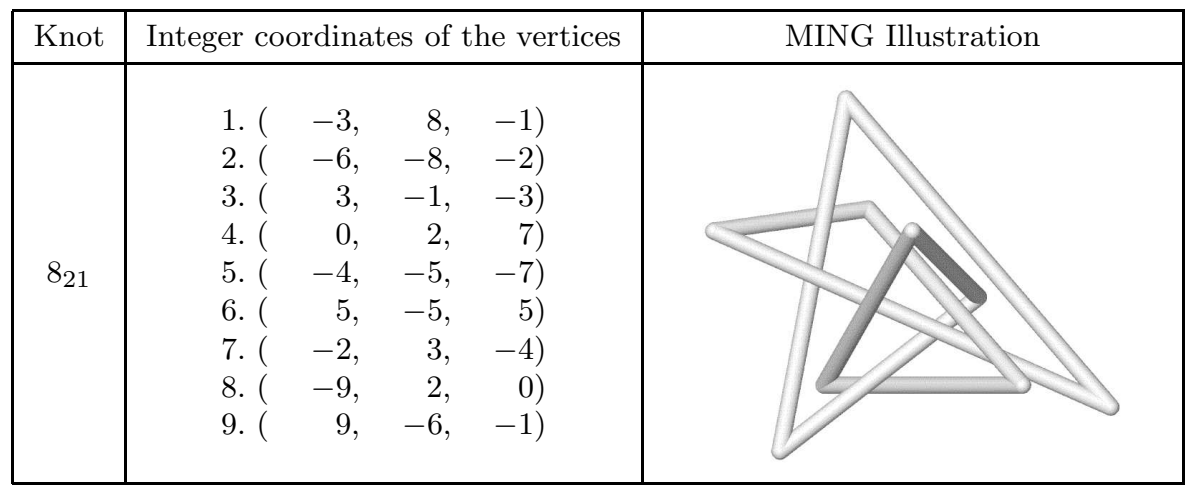

It should be noted that coordinates for some of these knots have previously appeared elsewhere. Knot $4_{1}$ appears in [9], knot $5_{2}$ in [4], and each of the knots $3_{1}, 4_{1}, 5_{2}$, and $6_{2}$ appear in [6].

In addition to the illustrations already provided in this paper, a more aesthetic result was also obtained. Based on the symmetry presentation of the figure- 8 knot $4_{1}$ which appeared in [2], a symmetry presentation was constructed using only 8 edges, as shown in the following table. It is called a symmetry presentation because a simple rotation of $90^{\circ}$ counter-clockwise yields its mirror image, a knot with the same projection but with its crossings reversed. This result is especially interesting because it shows that this figure- 8 knot and its mirror image are in the same path component of $M_{8}$ (hence too in $M_{n}$ for $n \geq 8$ ), where $M_{n}$ is the space of all knots with n edges [7].

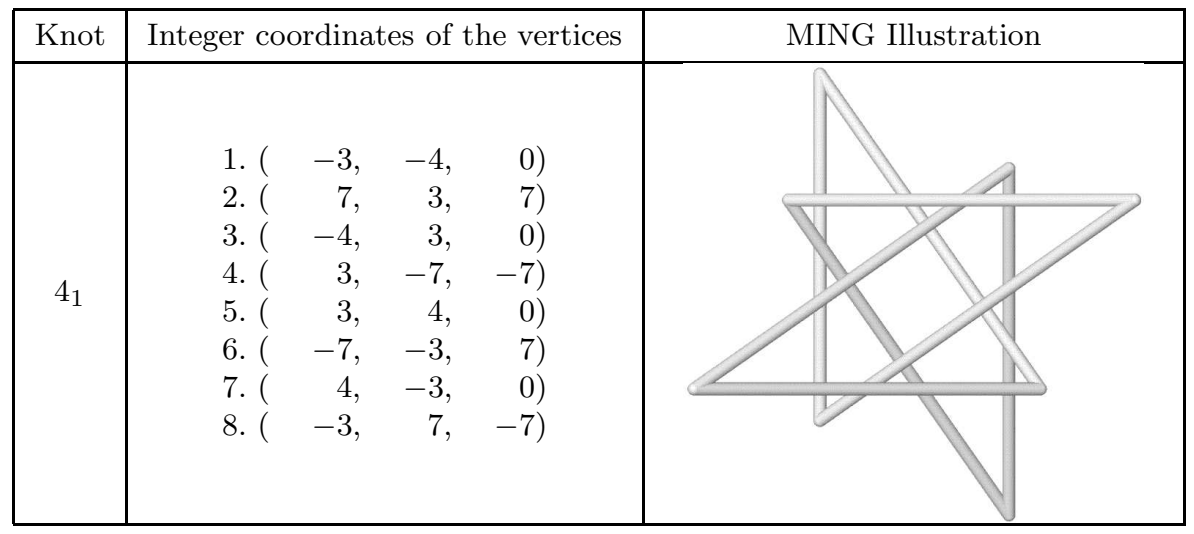

\section{References}

[1] C. C. Adams, B. M. Brennan, D. L. Greilsheimer and A. K. Woo, Stick numbers and compositions of knots and links, Journal of Knot Theory and Its Ramifications, to appear.

[2] E. Flapan, Rigid and non-rigid achirality, Pacific Journal of Mathematics, 129(1):57-66, 1987. 
[3] K. Hunt, KED, The University of Iowa. A computer program used to draw knots, http://www.cs.uiowa.edu/ hunt/knot.htm.

[4] G. T. Jin, Polygon indices and superbridge indices, Journal of Knot Theory and Its Ramifications, to appear.

[5] G. T. Jin and H. S. Kim, Polygonal knots, Journal of the Korean Mathematical Society, 30(2):371-383, 1993.

[6] K. C. Millett, Knotting of regular polygons in 3-space, in K. C. Millett and D. W. Sumners, editors, Random Knotting and Linking, pages 31-46. World Scientifis, Singapore, 1994.

[7] R. Randell, Invariants of piecewise-linear knots, this volume.

[8] D. Rolfsen, Knots and Links, Publish or Perish, Inc., Houston, 1990.

[9] K. Smith, Generalized braid arrangements and related quotient spaces, $\mathrm{PhD}$ thesis, The University of Iowa, Iowa City, IA, USA, 1992.

[10] Y-Q. Wu, MING, The University of Iowa, Iowa City, Iowa. A computer program used to draw knots, available via anonymous ftp at ftp.math.uiowa.edu/pub/wu/ming. 